\title{
Stability on turning of Inconel 718 using vibration-assisted machining
}

\author{
Matheus Coelho Cosenza ${ }^{1}$, Marcos Valério Ribeiro ${ }^{2}$, and Anna Carla Araujo ${ }^{1}$ \\ ${ }^{1}$ Mechanical Engineering Program - COPPE/UFRJ Universidade Federal do Rio de Janeiro \\ 2 Department of Materials and Technology - UNESP Guaratingueta Universidade Estadual Paulista
}

Abstract: Inconel is a hard-to-cut material that uses very low cutting speed. In turning operation added to the low cutting velocity, there is also a second limitation to productivity: chatter. In order to reduce chatter, the depth of cut is reduced and the material removal rate is lower. One option to avoid reducing the depth of cut is using vibration assistance machining. This paper presents a numerical investigation using $1 D$ vibrations in the feed direction in order to reduce chatter in turning Inconel 718.

Keywords: metal cutting, chatter, hard-to-cut materials, VAM

\section{INTRODUCTION}

As the aerospace, oil and gas and chemical industries advance, the need to improve and develop new manufacturing processes is a constant challenge. Researches and studies have been held to discover materials and methods to not only achieve high precision, but also to reduce the production time. Inconel and titanium alloys have excellent mechanical properties, nevertheless, as per its low thermal conductivity and high hardening, cutting speed ranges and tool life are below other metallic materials (Sharman et al., 2015). Furthermore, chatter is also a limitation on high production, caused by the friction between the tool and the workpiece (Urbicain et al., 2013). So, there are two main barriers in metal cutting against this progress: difficult-to-cut materials and chatter.

The vibration-assisted machining (VAM) is one method used for chatter mitigation, it adds small-amplitude and highfrequency displacement to the tool. Brehl and Dow (2008) presents the VAM benefits from the periodic separation between the tool rake face and the uncut material generated by the vibration to produce thinner chips, increase form accuracy and extend tool life. For 1D VAM, it is used a ultrasonic generator and a sonotrode (booster and horn) to create and amplify reciprocating harmonic motin of high frequency keeping a low amplitude. The tool is attached to the end of the horn and positioned so that the rake face is normal to the direction of vibration motion.

This paper propose a numerical simulation of chatter suppression using small amplitude tool displacement in the feed direction during turning Inconel 718. The goal is to analyze and compare the results with experiments previously published. Furthermore, the regions of instability are taken from a published paper and it is proposed to compare two situations in non-stable region, with and without small amplitudes, to confirm the benefits of VAM.

\section{MECHANICAL MODEL FOR DYNAMIC CUTTING FORCE}

Chatter is a self-excited vibration that can occur during machining operations that can be classified in two different categories, primary and secondary chatter (Quintana and Ciurana, 2011). The first one is caused by the friction between the workpiece and the cutting tool, by mode coupling and thermo-mechanical effects on the chip formation. The second and most relevant one, secondary chatter, can occur because most metal cutting operations contain overlapping cuts, which can generate a vibration amplification. As a result of the cutting tool vibration, the workpiece surface acquires a wavy surface and, consequently, the cutting force and the chip thickness change in relation to the difference between the uncut material left by the previous revolution and the current one. Basically, chatter can be avoid by estimating the stablility lobe diagram and adjusting the cutting parameters to maintain the system in the stable area. However, in the past years, a different approach have been used to manage the system vibration, this strategy uses elements, devices and actuators to modify the system behavior and control chatter.

\section{Chatter in turning operations}

Turning is a machining process in which the workpiece rotates around the $x$ axis, as shown in Figure 1a, with a $\Omega$ spindle speed, while the tool holder moves in the feed direction parallel to $x$. The feed velocity is a function of the feed per revolution $f_{r}$ and the spindle speed $\Omega$, which is calculated considering the cutting speed $V_{c}$ and the workpiece diameter $D$. As presented in Figure $1 \mathrm{~b}$, the depth of cut $a_{p}$ is the difference between the workpiece and the machined radius, if there is no vibration in the radial direction.

As shown in Figure 1a, if considering both tool and workpiece rigid, the chip would have a constant uncut chip thickness $h_{0}$, calculated as a function of the feed $f(\mathrm{~mm} / \mathrm{rev})$ and the lead angle $\kappa_{r}$. In this article it is considered that the 


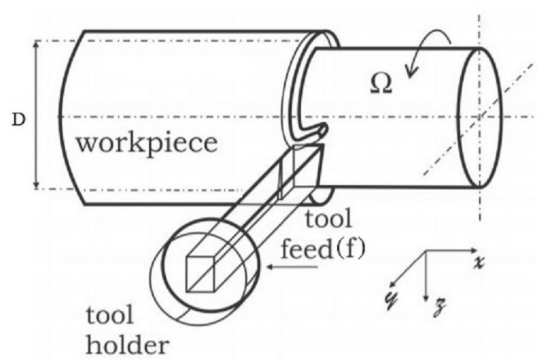

(a) Rigid tool

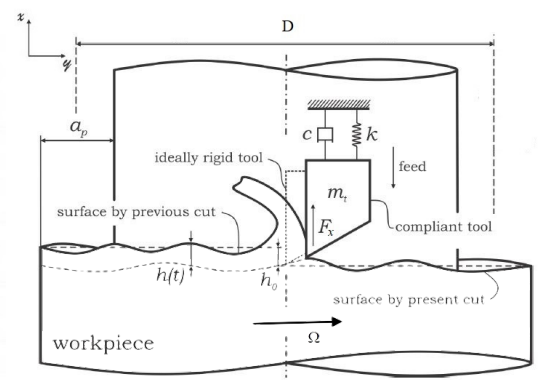

(b) Compliant tool

Figure 1 - Chip removal in orthogonal turning. (adap. from Hajdu et al. (2015))

cutting edge is orthogonal to the feed direction, so $\kappa_{r}=\pi / 2$ and $h_{0}=f_{r} \sin \kappa_{r}=f_{r}$.

In real conditions, components, like boring bars and tool holders, affect the flexibility of the system causing vibration and generating a non-uniform surface, as presented in Figure 1b. In this case, the tool tip position is moving as a result of the tool deflection $x(t)$. The uncut chip thickness is not constant $h(t) \neq h_{0}$ due to $x(t)$ and the workpiece surface machined in the previous revolution $x(t-\tau)$ :

$$
h(t)=h_{0}+x(t-\tau)-x(t)
$$

For a system that vibrates only in the feed direction, as shown in Figure 1b, the feed force $F_{x}(t)$ is a function as the specific cutting energy in feed direction $K_{x}$, depth of cut and the instantaneous uncut chip thickness $h(t)$ (Eq. 2). The cutting-force expoent $q$ has to be consider to evaluate the force in the vibration direction (Hajdu et al., 2015).

$$
F_{x}=K_{x} \cdot a_{p} \cdot h(t)^{q}
$$

Assuming the cutting tool is always in contact with the workpiece, in other words, the chip thickness is never less than zero, the system can be analyzed as a one-dimensional oscillator with excitation in the feed direction $\mathrm{x}$. Since instantaneous chip thickness $\mathrm{x}(\mathrm{t})$ depends on the workpiece surface machined in the past revolution $\mathrm{x}(\mathrm{t}-\tau)$ and considering the dynamical parameters: modal mass $m_{t}$, damping $c$, stiffness $k$ and the cutting parameter: feed velocity $v_{f}$, the governing equation can be written as:

$$
m_{t} \ddot{x}(t)+c \dot{x}(t)+k x(t)=F_{x}\left(K_{x}, a_{p}, h(t), q\right)=K_{x} a_{p}\left(v_{f} \tau(t)+x(t-\tau(t))-x(t)\right)^{q}
$$

The stability of the system can be analysed for each spindle speed $\Omega$. Figure 2 shows the diagram of stability lobes, which presents the depth of cut alim that bounds the values of depth cut that maintain the system stable and the values that result in instability and chatter.

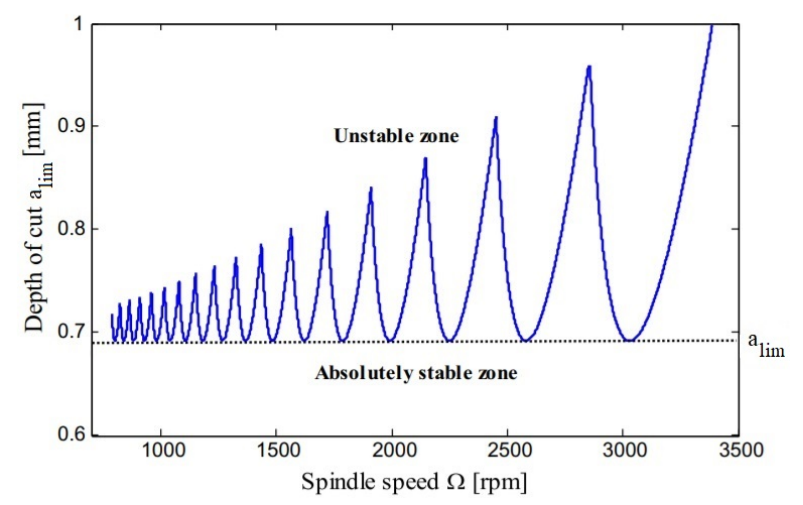

Figure 2 - Diagram of stability lobes (Lu et al., 2018).

\section{Vibration-assisted machining (VAM)}

Althought vibration is usually avoided, it can also be used to reduce cutting forces and produce thinner chips. The Vibration-assisted machining (VAM) adds a small amplitude with high frequency displacement to the cutting motion of the tool, generates an improvement in surface finish, form accuracy and burr reduction when compared with conventional machining. 


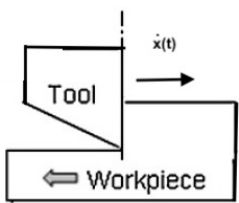

(a) $t=t_{1}$

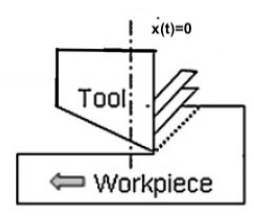

(b) $t=t_{2}$

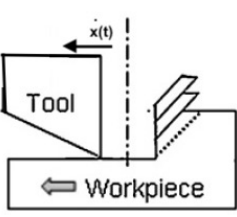

(c) $t=t_{3}$

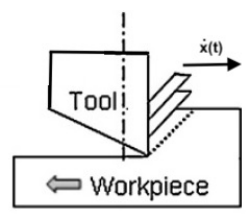

(d) $t=t_{1}^{\prime}$

Figure 3 - 1D vibration-assisted machining (adap. Brehl and Dow (2008))

The critical feed velocity is the one the rake face lose contact with the uncut material. For 1D VAM, the tool vibrates harmonically in a linear path in the feed direction, so $x(t)$ is known and controlled. In Fig. 3a, $t=t_{1}$, the tool has just started to cut the material and the velocity relative to the work $\dot{x}(t)$ is in the direction of $V_{f}$. In Fig. $3 \mathrm{~b}, t=t_{2}$, the tool already is though the vibration linear path, $\dot{x}(t)$ is equal to zero. After that, the tool goes slightly to reverse direction, in $t=t_{3}$ (Fig. 3c), and the tool lose contact with the workpiece. In Fig. 3d, $t=t_{1}^{\prime}$, the tool reestablish contact with the material to start a new cutting cycle with $\dot{x}(t)>0$ again.

Eq. 4 describes the feed controled position, where $\omega_{f}$ is the angular frequency, $V_{f}$ is the feed velocity and $A_{f}$ is amplitude of the tool vibration Brehl and Dow (2008). The critical feed velocity $V_{f}^{\text {lim }}$ that would cause the separation between the tool rake face and the workpiece surface is described in Eq. 6:

$$
\begin{aligned}
& x(t)=A_{f} \sin \left(\omega_{f} t\right)+V_{f} . t \\
& \dot{x}(t)=\omega_{f} A_{f} \cos \left(\omega_{f} \cdot t\right)+V_{f} \\
& V_{f}^{\text {lim }}=\quad \omega_{f} A_{f}
\end{aligned}
$$

When the velocity is greater or equal to the critical value, the tool is always in contact with the work surface. On the other hand, when the opposite happens, $V<V_{f}^{\text {lim }}$, the cutting is interrupted and the benefits of VAM can be noticed (better surface finish, form accuracy, etc.).

If one considers that VAM uncut chip thickness $h^{\prime}(t)$ will also change due to the vibration applied to the system (Eq. 7), the governing equation (Eq. 8) can be written as:

$$
\begin{aligned}
h^{\prime}(t)= & h(t)+A_{f} \sin \left(\omega_{f} t\right)=h_{0}+x(t-\tau)-x(t)+A_{f} \sin \left(\omega_{f} t\right)=v_{f} \tau(t)+A_{f} \sin \left(\omega_{f} t\right)+x(t-\tau(t))-x(t) \\
& \left.m_{t} \ddot{x}(t)+c \dot{x}(t)+k x(t)=F_{x} K_{x}, a_{p}, h(t), q\right)=K_{x} a_{p}\left(v_{f} \tau(t)+A_{f} \sin \left(\omega_{f} t\right)+x(t-\tau(t))-x(t)\right)^{q}
\end{aligned}
$$

\section{NUMERICAL SIMULATION}

The numerical simulation analyze the effects and benefits of VAM comparing the algorithm considering constant $h_{0}$ and VAM uncut chip thickness $h^{\prime}(t)$. Beyond that, the results will be compared with some experiments previously published to validate the routine. As proposed by (Altinas, 2000), first, a frequency greater than the natural frequency $f_{n}$ is chosen as the chatter frequency $f_{c}$ so $\omega_{c}>\omega_{n}$.

$$
\phi=\frac{X(s)}{F_{f}(s)}=\frac{\omega_{n}^{2}}{k_{x}\left(s^{2}+2 \xi \omega_{n} s+\omega_{n}^{2}\right)}
$$

Applying the Laplace Transformation, the transfer function is found (Eq. 9) and the phase angle $\psi$ can be written as:

$$
\tan (\psi)=\frac{\sin \left(\omega_{c} T\right)}{\cos \left(\omega_{c} T\right)-1}
$$

Giving that $r$ is the ratio between the chatter frequency and the natural frequency and $G\left(\omega_{c}\right)$ is the real part of the transfer function for the critical stability (Eq. 11), critical depth cut is given in Eq.12. Finally, the spindle speed $n(k)$ for each stability lobe $k=1,2,3, \ldots$ is calculated and the routine will repeat the procedure by scanning the chatter frequencies.

$$
\begin{array}{r}
G\left(\omega_{c}\right)=\frac{1-r^{2}}{k_{x}\left[\left(1-r^{2}\right)^{2}+(2 \xi r)^{2}\right]} \\
a_{l i m}=\frac{-1}{2 K_{x} G\left(\omega_{c}\right)} \\
n(k)=\frac{120 \pi f_{c}}{2 \psi+3 p i+2 k \pi}
\end{array}
$$




\section{Cutting parameters and dynamics conditions}

In order to compare the results obtained and the ones already published the routine must consider the same parameters used by Palacios et al. (2018). The workpiece is an cold worked Inconel 718 bar with 12.4 mm diameter, havind around $30 \mathrm{HC}$ hardness, and its behavior is studied near the depth cut limit $a_{\text {lim }}$.

Based on the results obtained by Palacios et al. (2018), the tool-holder have only one vibration mode with modal values of $f=1650 \mathrm{~Hz}, \zeta=0.0662$ and $m=0.2866 \mathrm{~kg}$. In addition, Palacios et al. (2018) also presented in Figure 4 the cutting conditions that produce a stable, unfilled circles, and unstable, triangles, scenario.

According to Brehl and Dow (2008), the operational VAM frenquecy is usually, approximately, $20 \mathrm{or} 40 \mathrm{kHz}$ and the amplitude magnitude is between 3 and $20 \mu \mathrm{m}$. If this boundary values are not respected, the tool vibration can become unstable, resulting in a possible impact damage to the cutting tool, poor surface finish and reduction of the cutting tool life. In simulation it is used $20 \mathrm{kHz}$ with $10 \mu \mathrm{m}$. The cutting conditions simulated are: (1) stable condition without VAM: $f_{z}=0.1(\mathrm{~mm} / \mathrm{rev})\left(V_{f}=40 \mathrm{~mm} / \mathrm{min}\right),(2)$ unstable condition without VAM: $f_{z}=0.6(\mathrm{~mm} / \mathrm{rev})\left(V_{f}=120 \mathrm{~mm} / \mathrm{min}\right)$ and (3) VAM applied to $f_{z}=0.6(\mathrm{~mm} / \mathrm{rev})\left(V_{f}=120 \mathrm{~mm} / \mathrm{min}\right)$. In all cases spindle Speed $\Omega=200 \mathrm{rpm}$.

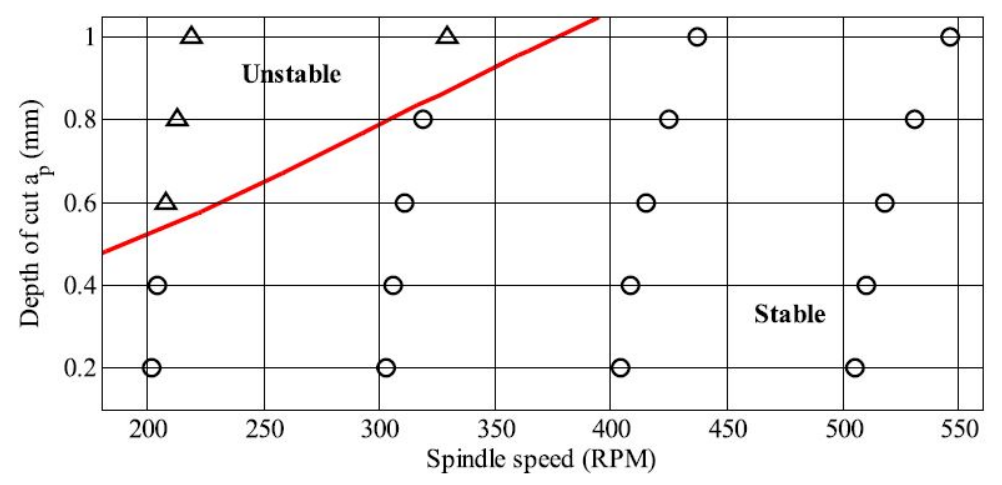

Figure 4 - Predicted stability obtained Enhanced Multistage Homotopy Pertubation (EMPHM) compared with experimental results for SNMG tool with $f_{z}=0.1 \mathrm{~mm} / \mathrm{rev}$. Palacios et al. (2018)

\section{REFERENCES}

Altinas, Y., 2000, Manufacturing Automation: metal cutting mechanics, machine tool vibrations, and CNC design. Cambridge University Press.

Brehl and Dow, 2008, Review of vibration-assisted machining. Precision Engineering, 32(3):153 - 172.

Hajdu, D., Insperger, T., and Stepan, G., 2015, Sensitivity of stability charts with respect to modal parameter uncertainties for turning operations. IFAC-PapersOnLine, 48(12):63 - 68.

Lu, K., Lian, Z., Gu, F., and Liu, H., 2018, Model-based chatter stability prediction and detection for the turning of a flexible workpiece.Mechanical Systems and Signal Processing, 100:814 - 826.

Palacios, J., Olvera, D., Urbikain, G., Elas-Ziga, A., Martnez-Romero, O., de Lacalle, L. L., Rodrguez, C., and MartnezAlfaro, H., 2018, Combination of simulated annealing and pseudo spectral methods for the optimum removal rate in turning operations of nickel-based alloys. Advances in Engineering Software, 115:391 - 397.

Quintana, G. and Ciurana, J., 2011, Chatter in machining processes: A review.International Journal of Machine Tools and Manufacture, 51(5):363 - 376.

Sharman, A., Hughes, J., and Ridgway, K., 2015, The effect of tool nose radius on surface integrity and residual stresses when turning inconel 718. J Mater Process Technol, 216:123-132.

Urbicain, G., Palacios, J., Fernandez, A., Rodriguez, A., de Lacalle, L. N. L., and Elias-Zuniga, A., 2013, Stability prediction maps in turning of difficult-to-cut materials. Procedia Engineering, 63:514 - 522.

\section{RESPONSIBILITY NOTICE}

The author(s) is (are) the only responsible for the printed material included in this paper. 